\title{
Alkaline Mineral Supplementation Decreases Pain in Rheumatoid Arthri- tis Patients: A Pilot Study
}

\author{
Regina Maria Cseuz ${ }^{1}$, Istvan Barna ${ }^{2}$, Tamas Bender ${ }^{3}$ and Jürgen Vormann*,4 \\ ${ }^{I}$ Revita Klinik, Budapest, Hungary; ${ }^{2}$ Institute of Experimental Medicine of the Hungarian Academy of Sciences, \\ Budapest, Hungary; ${ }^{3}$ Hospital Brothers of St. John of God, Budapest, Hungary, ${ }^{4}$ Institute for Prevention and Nutrition, \\ Ismaning, Germany
}

\begin{abstract}
The aim of this pilot study was to investigate the efficacy of an alkaline mineral supplement as a means of suppressing disease activity in rheumatoid arthritis (RA) patients, and to check whether any change occurs in the circulating beta-endorphin concentration. Thirty-seven patients with moderately active RA of at least two years duration, who were receiving stable pharmacological treatment, participated in a 12-week study. All patients were randomly allocated to a supplemented group (30g of an alkaline mineral supplement daily) or to an unsupplemented group. Their usual diet and medication was maintained. Disease activity, pain, and health-associated status were recorded (DAS 28 - Disease Activity Score 28, VAS - visual analogue scale for pain, HAQ - Health Assessment Questionnaire). Plasma immunoreactive endorphin (ir-EP) was measured in the study groups and also in healthy subjects. DAS 28 and VAS decreased in the supplemented group, whereas there was no change in these parameters during the trial in the control group. The functions (HAQ) of the supplemented patients improved. The ir-EP levels increased in both groups but to a higher degree in the supplemented group. During the trial, medication (NSAIDs and steroids) could be reduced in the supplemented group only. Conclusion: This study suggests that an alkaline supplement may improve function and pain in rheumatoid arthritis and may represent an easy and safe addition to the usual treatment of RA patients.
\end{abstract}

Keywords: Pain, rheumatoid arthritis, acid-base balance, beta-endorphin.

Conference Paper, presented at the $2^{\text {nd }}$ International Acid-Base Symposium, Nutrition - Health - Disease in Munich, September 2006.

\section{INTRODUCTION}

A large body of epidemiological and experimental studies has demonstrated that nutrition has an important impact on the occurrence and severity of various chronic diseases [1]. The level of disease-associated pain may also be dietrelated. For example, patients with rheumatoid arthritis (RA) frequently experience a positive effect of a change in diet on the activity of their disease. The hypothesis of our present study was that patients with RA, consuming an ordinary Western diet as most of the population of the world's developed countries, develop a diet-induced low-grade systemic metabolic acidosis [2]. There is strong evidence that a diet rich in fruit and vegetables acts protectively against a wide variety of human diseases. However, such a diet usually also supplies excess alkalinity, and part of its beneficial effects might be associated with a reduction of the chronic acid load [3]. The chronic inflammatory process in RA patients leads to a local increase in acidity; the $\mathrm{pH}$ in synovial fluids from patients with RA is significantly lower than that in patients with osteoarthritis or controls [4]. This change in local acidity might aggravate pain symptoms, especially in connective tissues. Therefore, alkaline supplementation might lead to an improvement of the clinical outcome in RA patients. In patients with chronic low back pain, alkaline supplementa-

*Address correspondence to this author at the Institute for Prevention and Nutrition, Adalperostr. 37, D-85737 Ismaning, Germany; Tel: +49 (0)89 55267989; Fax: +49 (0)89 55267990; E-mail: vormann@ipev.de tion significantly reduces pain and disability [5]. Our own observations of some RA patients also suggest the positive effect of a complementary treatment with alkalines. We have therefore conducted a pilot study to investigate, in more detail, the effects of an alkaline supplement on pain symptoms in patients with RA. In addition to subjective indicators of pain, disability, and quality of life, the change of the concentration of beta-endorphin (ir-EP) has been determined in the plasma of patients as a more objective parameter of pain problems. Significantly lower ir-EP levels have been reported in RA patients than in controls, and an inverse correlation has been found between the rheumatoid disease activity score and plasma ir-EP concentration [6].

\section{MATERIALS AND METHODS}

\section{Patients and Study Design}

Prior to commencing the study, approval by the local ethical committee (Scientific Committee of St John's Hospital, Budapest) was obtained, and the ethical principles of the Helsinki Declaration were followed. All patients were informed orally and in writing about the study design and the underlying hypothesis and of the participant's right to withdraw at any time. The study design was a single-center randomized parallel trial over a period of three months.

\section{Patients}

Out of 76 outpatient candidates who were screened for the trial and were willing to participate, 37 patients fulfilled 
all the inclusion criteria according to Table $\mathbf{1}$. These patients were randomly selected into a supplemented group or an unsupplemented control group. Baseline characteristics of patients in both groups are shown in Table 2.

\section{Supplementation}

After a 4-week wash-out period (other supplements), patients in the supplemented group started to take, on a twice

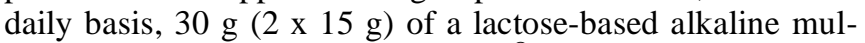
timineral supplement (Basica Vital ${ }^{\circledR}$, Protina Pharmaceuti- cals, Germany) for a 12-week period in addition to their usual medication. The mineral composition was as follows (mg/daily dose): Ca (400), K (250), Na (250), Mg (100), Fe (5), $\mathrm{Cu}(1)$ all as citrates, $\mathrm{Zn}(5)$ as gluconate, I (0.1) as potassium iodide, Mo (0.08) as sodium molybdate, $\mathrm{Cr}(0.06)$ as chromium chloride, $\mathrm{Se}(0.03)$ as sodium selenite. The alkaline mineral supplement was taken twice daily in the form of a powder mixed in one of the following: soup, yoghurt, tea, or cereal. A suitable inert placebo in a similar daily dose was not available, and the use of sugar as a placebo seemed not

Table 1. Inclusion and Exclusion Criteria

\section{Exclusion Criteria:}

- Co-morbidity (such as diabetes, gastrointestinal diseases) - except hypertension and osteoporosis.

Table 2. Baseline Characteristics of Patients who Completed the Trial. Data are Presented as Mean (Range) Unless Otherwise Stated

\begin{tabular}{|c|c|c|}
\hline & SD Patients (n=19) & CG Patients $(n=17)$ \\
\hline Body Mass Index $\left(\mathrm{kg} / \mathrm{m}^{2}\right)$ & $26.1(22.3-31.9)$ & $27.6(17.6-49.5)$ \\
\hline Disease Duration (years) & $11.8(2-26)$ & $12.8(2-22)$ \\
\hline DAS28, Score & $5.2(3.6-6.9)^{\dagger}$ & $4.5(2.8-6.0)$ \\
\hline ARA Class I* & 0 & 0 \\
\hline Class II & 5 & 5 \\
\hline Class III & 14 & 11 \\
\hline NSAID & 19 & 17 \\
\hline Corticosteroids/mg prednisolone & $6 / 4.6$ & $7 / 4.2$ \\
\hline \multicolumn{3}{|l|}{ Co-morbidity } \\
\hline Hypertension & $2 / 2$ & $4 / 4$ \\
\hline Osteoporosis & $1 / 0$ & $3 / 3$ \\
\hline
\end{tabular}

* Number of patients.

$\dagger$ Statistically significant difference between SD and CG, $\mathrm{p}<0.05$.

Abbreviations used in Table 2. : SD - supplementary diet; CG - control group; DAS - disease activity score; ARA -American Rheumatology Association; NSAID - non-steroidal anti-inflammatory drug, The use of other disease modifiing drugs was equally distributed over both groups. 
to be appropriate. Therefore, a placebo was omitted in this pilot trial. During the trial, patients were not allowed to take any dietary supplements except for the alkaline mineral supplementation in the supplemented group. The individual dose of non-steroidal anti-inflammatory drugs (NSAID) could be adjusted but had to be recorded.

\section{Determinations and Measurements}

All patients were evaluated by the same investigator in accordance with a written protocol that included medical history, ACR criteria [7], complete evaluation, articular evaluation, and extra articular evaluation of RA (history of rheumatoid nodules, Reynard's phenomenon, or pulmonary, cardiac, dermal, ocular, and nervous system involvement). Disease activity was assessed by determination of the number of swollen joints, the score of tender and swollen joints, and the duration of morning stiffness (in minutes). A composite disease activity score (DAS 28) [8], a physical function index Health Assessment Questionnaire (HAQ) [9], Creactive protein (CRP), and rheumatoid factor were measured at baseline and 4, 8, and 12 weeks after starting the trial. DAS28 is a composite disease activity index and also a response index with good discriminatory validity. It includes 28 joint counts for tenderness (tender joint count) and swelling (swollen joint count), the erythrocyte sedimentation rate (ESR), and the patient's global assessment of disease activity on a horizontal visual analog scale (patient global VAS, 0$100 \mathrm{~mm}$ ). As a parameter connected to pain, the level of irEP was determined in blood samples of both patient groups and from healthy subjects ( 6 females, 6 males; mean age: 45 years). Blood samples were collected into $\mathrm{K}_{2}$-EDTAcontaining plastic tubes and then centrifuged, after which plasma aliquots were stored at $-20{ }^{\circ} \mathrm{C}$ until assayed. Details of the EP radioimmune assay (RIA) including the percental cross-reaction data were as described earlier [10]. In short, synthetic human EP (Sigma) was used both for the standard and ${ }^{125}$ I-labeled tracer, and a second antibody was used to separate the bound and free fractions.

\section{Compliance}

Compliance of supplemented patients was monitored by the weekly determination and recording of the $\mathrm{pH}$ of the first morning urine with $\mathrm{pH}$ paper strips by the patients themselves. Supplementation induced a significant increase in urinary $\mathrm{pH}$ by at least one $\mathrm{pH}$ unit after one week in all supplemented patients and remained high throughout the supplementation period thus indicating adherence to the supplement.

\section{Statistical Methods}

Determined parameters were normally distributed and variations were compared between time 0 and 4, 8, and 12 weeks, respectively, and between the supplemented and unsupplemented groups by using Student's t-test. Frequencies of reduction of medication were compared between groups by means of the Fisher exact test. All reported $\mathrm{P}$ values are 2-sided.

\section{RESULTS}

A total of 37 patients were enrolled of whom 19 were randomly allocated to the supplemented group and 18 to the unsupplemented control group. In one control patient, an intra-articular injection with triamcinolone hexacetonide was reported what expectedly influenced the disease acitivity, and this patient was excluded from the study. Hence, 19 supplemented and 17 control patients completed the trial. At the start of the experiment, the two groups were equal in all respects, except for disease activity score (DAS28). At baseline, the control patients showed a score of 4.5 (range: 2.8$6.0)$ versus the supplemented group with a score of 5.2 (range: 3.6-6.9); this difference is statistically significant ( $t$ test). At the end of the observation period, the supplemented group, in which members had started with significantly higher disease activity score, showed significantly lower DAS28 compared with control patients (Fig. 1). DAS28 decreased in the supplemented group, whereas there was no significant change in disease activity score during the trial in the control patients. There were also significant differences between supplemented and unsupplemented groups at 4,8 , and 12 weeks. The level of pain (according to the patient's visual analog scale (VAS)) decreased to a considerable extent in the supplemented group (Fig. 2). In the control patients, the pain increased between week 0 and week 4 . Be-

disease associated symptom index (DAS28)

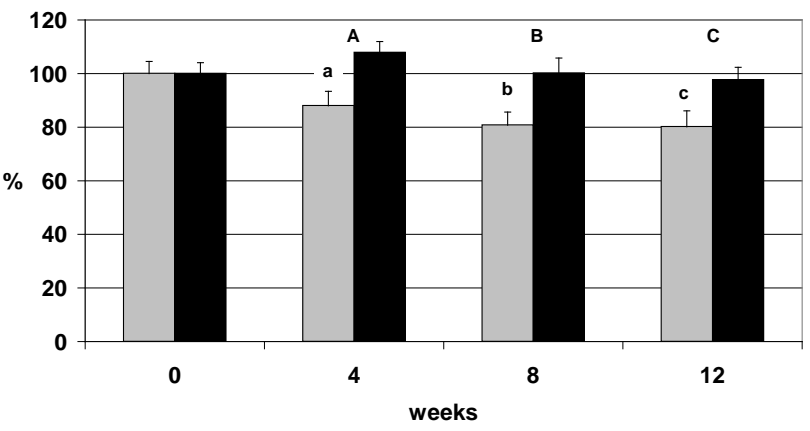

Fig. (1). DAS28 in supplemented (first column) and unsupplemented (second column) RA patients at $0,4,8$, and 12 weeks. Significant difference in comparisons with time 0 of the respective group; $a: p=0.049 ; b: p=0.011, c: p=0.004$. Significant differences between groups; $\mathrm{A}: \mathrm{p}=0.006 ; \mathrm{B}: \mathrm{p}=0.016, \mathrm{C}: \mathrm{p}=0.028$; mean \pm SEM.

pain (VAS)

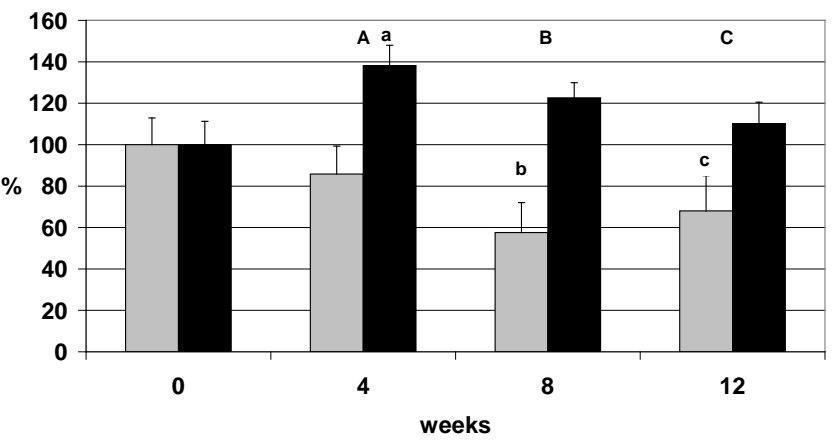

Fig. (2). Pain level according to a visual analog scale in supplemented (first column) and unsupplemented (second column) RA patients at $0,4,8$, and 12 weeks. Significant difference in comparisons with time 0 of the respective group; $a: p=0.045 ; b: p=0.004$, $\mathrm{c}: \mathrm{p}=0.048$. Significant differences between groups; $\mathrm{A}: \mathrm{p}=0.003$; $\mathrm{B}: \mathrm{p}=0.001, \mathrm{C}: \mathrm{p}=0.037$; mean \pm SEM 
tween groups, significant differences were detected after 4, 8 , and 12 weeks. HAQ results showed a significant improvement in supplemented patients by the end of the trial, whereas no change was seen in the control patients (Fig. 3). At the end of the trial, the difference between groups also became significant.

physical function index (HAQ)

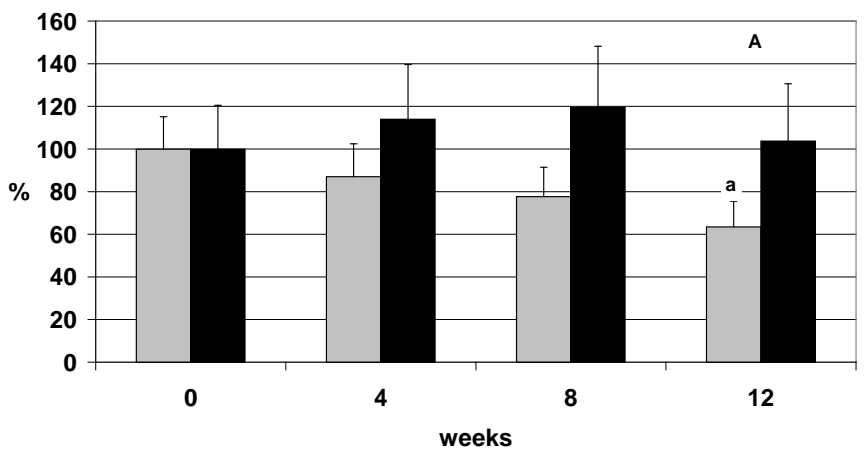

Fig. (3). Physical function index (HAQ) in supplemented (first column) and unsupplemented (second column) RA patients at 0,4 , 8 , and 12 weeks. Significant difference in comparisons with time 0 of the respective group; a: $p=0.009$. Significant differences between groups; $\mathrm{A}: \mathrm{p}=0.049$; mean \pm SEM.

ESR, CRP, and the level of rheumatoid factor showed no significant change during the trial in either of the groups (not shown).

In healthy subjects, the plasma ir-EP levels were significantly higher than in RA patients: (plasma ir-EP in fmol/ml, mean \pm SEM) healthy subjects, $12.6 \pm 3.9(\mathrm{n}=12)$; RA patients, $4.1 \pm 0.5(n=37)$. Plasma ir-EP levels significantly increased in the supplemented and control group; however, the increase in the supplemented group occurred earlier and was higher than in the control patients (Fig. 4); at the end of the supplementation period, there was also a significant difference between both groups.

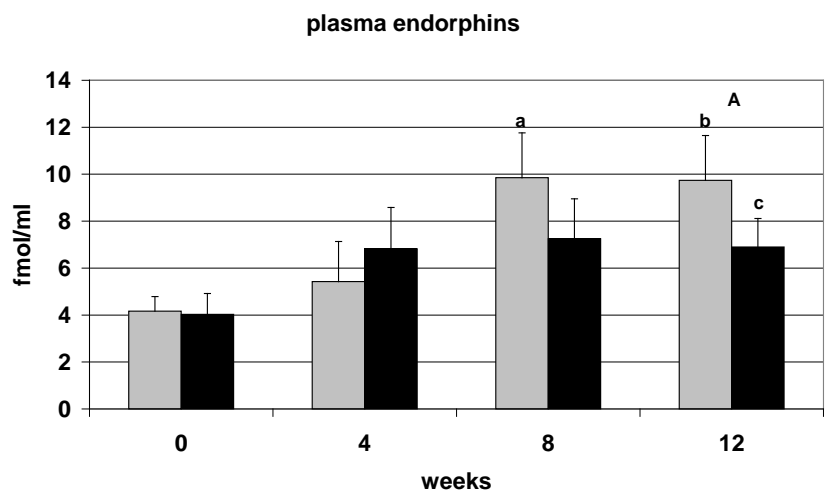

Fig. (4). Plasma endorphin concentration in supplemented (first column) and unsupplemented (second column) RA patients at 0,4 , 8 , and 12 weeks. Significant difference in comparison with time 0 of the respective group; $\mathrm{a}: \mathrm{p}=0.016 ; \mathrm{b}: \mathrm{p}=0.018, \mathrm{c}: \mathrm{p}=0.045$. Significant differences between groups; A: $p=0.043$; mean \pm SEM.

At the end of the study, the medication of 6 out of 19 supplemented patients was reduced: in 3 patients, the daily steroid dose was decreased by $2-4 \mathrm{mg}$, and three other pa- tients no longer needed to take NSAIDs. In the control group, one patient had to be put on medication because of hypertension during the trial, and one patient's steroid dosage had to be increased, in the other patients medication was unchanged. The reduction in medication in the supplemented compared with the control group was statistically significant according to the Fisher's exact test $(\mathrm{p}=0.014)$.

\section{DISCUSSION}

The cause of RA is still unknown, but it is likely to involve both genetic susceptibility and environmental factors such as diet [11]. The role of nutrition should be clarified with respect to two fundamental aspects: 1) Does it have any effect in the clinical expression of the disease or in susceptibility to RA? 2) Could any diet or nutrient supplementation play a role in the management of RA by alleviating symptoms such as pain, by decreasing the progression of the disease, or by reducing the reliance on or combating the sideeffects of NSAIDs ? [12].

Case-controlled studies indicate that the lifelong consumption of fish, olive oil, and cooked vegetables may have protective effects on the development or severity of RA [13, 14]. Patients with RA have been reported to consume too much total fat and too little polyunsaturated folic acid (PUFA) and fibre [15]. The so-called Western diet (which is also ingested by most of the population in Hungary) is well known to lead to the development of latent metabolic acidosis [16]. Compensation of this acidosis is possible by increasing the intake of organic mineral salts either from the diet (increased intake of vegetables and fruits) or from supplements. The main alkaline substances in our diet are citrates; a useful supplement therefore should contain a mixture of various citrate salts of sodium, potassium, calcium, and magnesium. The used supplement contains all these salts, together with trace elements and lactose, which increases the bioavailability of minerals. The used dose of the supplement provides a total of $45 \mathrm{mEq}$ base per day. The usual daily surplus of acid in the Western diet is $60 \mathrm{mEq}$ in an elderly population [17], and so a significant reduction in acid load is achieved with the supplementation. There is to date no indication that single constituents of the supplement alone work in improving the symptoms in RA patients.

Supplementation of the usual diet with alkaline minerals improved the DAS28 in this study. Of the contributory factors of DAS, the level of pain changed most remarkably in the supplemented group; however, laboratory parameters indicating the degree of inflammation (ESR, CRP) did not change in either of the groups, indicating that the severity of the disease by itself was not influenced by the supplementation. One might argue that plasma parameters of acid-base status ( $\mathrm{pH}$ and bicarbonate values) should have been determined in this study; however, these parameters are extremely well buffered, and changes in these parameters are much smaller than the probable $\mathrm{pH}$ or buffer changes in the interstitium [18].

The reduced pain sensation can be explained by the significant elevation of plasma ir-EP levels following alkaline supplementation. The exact physiological role of circulating ir-EP is still obscure. What is clear from biochemical studies is that ir-EP binds predominantly to the mu opioid receptors [19] that are present in several peripheral tissues including 
immune cells [20,21] and the synovial membrane [22]. Sporadic experimental data suggest that peripheral ir-EP and its opioid receptor system play a role in the physio-pathology of local inflammatory processes. The above experimental results and the reported negative correlation between RA activity and plasma ir-EP levels [23], together with the general opinion that relative ir-EP deficiency may play a part in the manifestation of autoimmune diseases [24, 25], all suggest a causality between the circulating ir-EP levels and the arthritis-induced pain.

In addition to a pain-reducing direct effect of endorphins, an alternative explanation might be the more alkaline environment in the local surrounding of the joints, as pain receptors are sensitive to small $\mathrm{pH}$ changes. Significant changes in $\mathrm{pH}$ have been shown to occur in the interstitium without detectable changes in blood $\mathrm{pH}$ [18].

The finding that 6 out of 19 patients taking an alkaline supplement could reduce their daily steroid or NSAID dosage also supports the view that a reduction in latent metabolic acidosis has a positive effect on pain sensation. As the chronic need for pain medication is connected to potential side-effects, it is of great importance to find safe adjuvant therapies for these patients, such as supplementation with alkaline minerals, even though the supplementation does not influence the severity of the disease itself.

The limitations of this study include its small sample size and the lack of an active control group. However, the results of this pilot trial should encourage further randomized placebo-controlled studies to investigate the effects of alkali therapy.

\section{CONCLUSIONS}

Studies of the effects of dietary habits and nutrient supplementation on RA are, to quote Ollier et al. [26], "hampered by the inherent variability in the clinical course of the disease and the wide spectrum of clinical phenotypes." Patients frequently self-prescribe complementary medicines including diet modifications. This leads to considerable difficulties in selecting an appropriate group of patients for study. Improvement in symptoms may be dependent on the severity of the disease and on the underlying inflammatory status. An important finding of this study is the significant decrease in pain and DAS28 level and elevation in plasma irEP levels following several weeks of alkaline supplementation. Our study is the first to demonstrate the effect of the metabolic acid-base balance on plasma ir-EP levels. The results of our study suggest that an alkaline supplement may improve function and pain in rheumatoid arthritis and represents an easy and safe addition to the usual treatment of RA patients.

\section{ACKNOWLEDGMENTS}

The authors wish to express their gratitude to all the patients who so willingly participated in the study. For her untiring help and painstaking administrative skills, Agnes Besenyei deserves extra special appreciation. We are also grateful to Professor George Kunos (U.S.National Institutes of Health) for his constructive remarks. Dr Beatrix Kocsis is warmly thanked for her constant and valuable support with the biochemistry. The authors also wish to express their thanks to Istvan Ratkó for assistance with the bio-statistics.
We are grateful to Protina Pharm GmbH for their financial support and for providing the mineral supplement. JV is a research consultant to Protina Pharm $\mathrm{GmbH}$ in acid-base metabolism. The other authors declare no competing interests.

\section{REFERENCES}

[1] Neel JV. When some fine old genes meet a new environment? In: Simopoulus A Ed, Evolutionary aspects of nutrition and health, Karger: Basel 1999; 1-15.

[2] Vormann J, Remer T. Dietary, metabolic, physiologic, and diseaserelated aspects of acid-base balance: foreword to the contributions of the second International Acid-Base Symposium. J Nutr 2008; 138: 413S-4S.

[3] Vormann J, Goedecke T. Acid base homeostasis. Latent acidosis as a cause of chronic disease. Schweiz Zschr Ganzheits Medizin 2006; 18: 255-66.

[4] Farr M, Garvey K, Bold AM, Kendall MJ, Bacon PA. Significance of the hydrogen ion concentration in synovial fluid in rheumatoid arthritis. Clin Exp Rheumatol 1985; 3: 99-104.

[5] Vormann J, Worlitschek M, Goedecke T, Silver B. Supplementation with alkaline minerals reduces symptoms in patients with chronic low back pain. J Trace Elem Med Biol 2001; 15: 179-83.

[6] Elbeialy A, Elbarbary M, Kamel M. Peripheral beta-endorphin in rheumatoid Arthritis. A correlation with the disease activity. Scand J Rheumatol 1997; 26: 88-91.

[7] Arnett FC, Edworthy SM, Bloch DA, et al. The American Rheumatism Association 1987 revised criteria for the classification of rheumatoid arthritis. Arthritis Rheum 1988; 31: 315-24.

[8] Van Riel PLCM, van Gestel AM. Clinical outcome measures in rheumatoid Arthritis. Ann Rheum Dis 2000; 59 (Suppl 1): 28-31.

[9] Bruce B, Fries J. The Stanford Health Assesment Questionnaire (HAQ): A review of its history, issues, porgress and documentation. J Rheumatol 2003; 30: 167-78.

[10] Barna I, Koenig JI. Effects of mediobasal hypothalamic lesion on immunoreactive ACTH/beta-endorphin levels in cerebrospinal fluid, in discrete brain regions, in plasma, and in pituitary of the rat. Brain Res 1992; 593: 69-76.

[11] Pattison DJ, Harrison RA, Symmons PMD. The role of diet in susceptibility to rheumatoid arthritis: a systematic review. J Rheumatol 2004; 31: 1310-9.

[12] Rennie KL, Hughes J, Lang R, Jebb SA. Nutritional management of rheumatoid arthritis: a review of the evidence. J Hum Nutr Diet 2003; 16: 97-109.

[13] Haugen M, Fraser D, Forre $\varnothing$. Diet therapy for the patient with rheumatoid arthritis. Rheumatology 1999; 38: 1039-44.

[14] Linos A, Kaklamani VG, Kaklamani E, et al. Dietary factors in relation to rheumatoid arthritis: a role for olive oil and cooked vegetables? Am J Clin Nutr 2000; 71: 1010.

[15] Calder PC, Zurier RB. Polyunsaturated fatty acids and rheumatoid arthritis. Curr Opin Clin Nutr Metab Care 2001; 4: 115-21.

[16] Cordain L, Eaton SB, Sebastian A, et al. Origins and evolution of the Western diet: health implications for the 21 st century. Am J Clin Nutr 2005; 81: 341-54.

[17] Rylander R, Remer T, Berkemeyer S, Vormann J. Acid-base status affects renal magnesium losses in healthy, elderly persons. J Nutr 2006; 136: 2374-7.

[18] Street D, Nielsen J-J, Bangsbo J, Juel C. Metabolic alkalosis reduces exercise-induced acidosis and potassium accumulation in human skeletal muscle interstitium. J Physiol 2005; 566: 481-9.

[19] Mizoguchi H, Tseng LF, Suzuki T, Sora I, Narita M. Differential mechanism of G-protein activation induced by endogenous muopioid peptides, endomorphin and betaendorphin. Jpn J Pharmacol 2002; 89: 229-34.

[20] Chan TK, Killam Jr KF, Chuang LF, et al. Mu opioid receptor gene expression in immune cells. Biochem Biophy Res Commun 1995; 216: 922-30.

[21] Rogers TJ, Peterson PK. Opioid G protein-coupled receptors: signals at the crossroads of inflammation. Trends Immunol 2003; 24 : 116-21.

[22] Hayashi K, Sugisaiki M, Ota S, Tanabe H. mu-Opioid receptor mRNA expression and immunohistochemical localization in the rat temporomandibular joint. Peptides 2002; 23: 889-93. 
[23] Martinez JH, Mondragon CE, Cespedes A. An evaluation of the antiinflammatory effects of intraarticular synthetic beta-endorphin in the canine model. Anest Analg 1996; 82: 177-81.

[24] Morch H, Pedersen BK. Beta-endorphin and the immune system possible role in autoimmune diseases. Autoimmunity 1995; 21: $161-71$.
Sacerdote P, Gaspani L, Panerai AE. Role of b-endorphin in the modulation of immune responses: perspectives in autoimmune diseases. Adv Exp Med Biol 2001; 63: 1037-41.

[26] Ollier WE, Harrison B, Symmons D. What is the natural history of rheumatoid arthritis? Best Pract Res Clin Rheumatol 2001; 15: 2748 .

(C) Cseuz et al.; Licensee Bentham Open.

This is an open access article licensed under the terms of the Creative Commons Attribution Non-Commercial License (http://creativecommons.org/licenses/by-nc/3.0/) which permits unrestricted, non-commercial use, distribution and reproduction in any medium, provided the work is properly cited. 